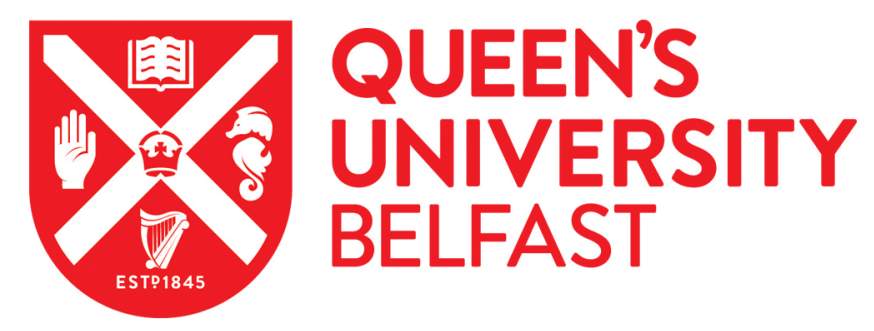

\title{
Measurements of Rainfall Rates From Videos
}

Dong, R., Liao, J., Li, B., Zhou, H., \& Crookes, D. (2017). Measurements of Rainfall Rates From Videos. In 10th International Congress on Image and Signal Processing, BioMedical Engineering and Informatics (CISP-BMEI 2017): Proceedings Institute of Electrical and Electronics Engineers Inc..

\section{Published in:}

10th International Congress on Image and Signal Processing, BioMedical Engineering and Informatics (CISPBMEI 2017): Proceedings

\section{Document Version:}

Peer reviewed version

\section{Queen's University Belfast - Research Portal:}

Link to publication record in Queen's University Belfast Research Portal

\section{Publisher rights}

(C) 2017 IEEE.

This work is made available online in accordance with the publisher's policies. Please refer to any applicable terms of use of the publisher.

\section{General rights}

Copyright for the publications made accessible via the Queen's University Belfast Research Portal is retained by the author(s) and / or other copyright owners and it is a condition of accessing these publications that users recognise and abide by the legal requirements associated with these rights.

Take down policy

The Research Portal is Queen's institutional repository that provides access to Queen's research output. Every effort has been made to ensure that content in the Research Portal does not infringe any person's rights, or applicable UK laws. If you discover content in the Research Portal that you believe breaches copyright or violates any law, please contact openaccess@qub.ac.uk. 


\title{
Measurements of Rainfall Rates From Videos
}

\author{
Rong Dong ${ }^{1}$, Juan $\mathrm{Liao}^{2}$, Bo Li ${ }^{2}$, Huiyu Zhou ${ }^{3}$, Danny Crookes ${ }^{3}$ \\ ${ }^{1}$ School of Electronics and Information, Nantong University, Nantong, China \\ ${ }^{2}$ School of Electronic Science and Engineering, Nanjing University, Nanjing, China \\ ${ }^{3}$ School of Electronics, Electrical Engineering and Computer Science, Queen's University Belfast, Belfast, United Kingdom
}

\begin{abstract}
Measuring rainfall rates from videos is a novel research topic. Due to rain motion, reflection of light and background clutter, it is extremely challenging to obtain accurate measurements. In this paper, we propose a new technique for measuring rainfall rates from videos, which consists of the following technical steps: first, we detect raindrops in an image using gray-tone functions and direction of rain streaks; we then select the focused raindrops, based on two features: average color tensor response and average intensity difference. Afterwards, the size of the raindrops is estimated and a raindrop size distribution (RSD) curve is created according to the use of the RSD in meteorology. Finally, a rainfall rate is obtained by fitting the RSD curve with a Gamma distribution model. In the experiment section presented in this paper, the proposed algorithm is evaluated under different light, moderate and heavy rainy conditions. The measurement results of the proposed algorithm are consistent with those of a can-type rain gauge.
\end{abstract}

Keywords- color tensor; logarithmic image processing; rain detection; rainfall rate; raindrop size distribution

\section{INTRODUCTION}

Measurement of rainfall rates are very important for climate analytics, water resources utilization and flood disaster prevention. Many types of instruments or methods for rainfall rates measuring are available nowadays.

Generally, they can be divided into manual, mechanical, microwave-based and optical categories. The widely used cantype rain gauge is a manual and conventional pluviometer. Though it is very simple and non-automatic, it has been and still used at many meteorological stations in the world. In the mechanical category, tipping-bucket rain gauge or electromechanical instruments [1][2] is commonly used. In the microwave-based category, weather radar is widely used to estimate precipitation [3-6]. In the optical category, laser systems or cameras are used. Laser-based systems [7-9] measured rain rates by detecting the coherence degradation of a laser beam caused by propagating through rain. The best known camera-based rain gauge system is the $2 \mathrm{D}$-VideoDistrometer (2DVD) which uses two high speed line scan cameras and background illumination [10][11]. Besides the line scan cameras, matrix cameras have also been used in the measurement of meteorological parameters [12-15]. All of those camera-based system require a specialized illumination component.

As an alternative to the above methods, in this paper we propose to measure rainfall rates from CCTV cameras. As CCTV cameras become more and more popular, such a rainfall measurement method is an economic and easily maintainable technique with high temporal resolution. In spite of its promise, however, as a Video Based Measurement (VBM) [16], video based rainfall rate measurement techniques had to deal with two major problems: (1) Image analysis: to accurately detect raindrops from videos and (2) Measurement: to establish a correct relationship between the quantities of the detected rain pixels and the corresponding rain rates.

To handle the first problem, we take advantage of a rain detection algorithm. Raindrops have complicated effects on images. To date, many research studies have been conducted in order to solve this challenging problem, e.g. [17-26]. All of them detect raindrops use arithmetic operations for real numbers. In this paper, we propose to detect raindrops in a Logarithmic Image Processing (LIP) framework. In terms of the second problem, we need to know the actual size of a rain streak detected on the image in order to calculate rainfall rates. Unfortunately, the 3D depth of a rain streak is hard to obtain and consequently the real size of the corresponding raindrop cannot be computed without this depth information. One of the solutions is to properly adjust the parameters of the used camera so that all the focused rain streaks are nearly at the same depth (i.e. at the position of the known focal plane). Afterwards, the size of the re-focused rain streaks can be calculated using an affine transformation based approach. To achieve this target, focused and defocused raindrops must be discriminated appropriately beforehand. In the literature, there is very little research work on this discrimination problem. Saylor and Sivasubramanian [14] used the existence of a bright hole in the center of a raindrop to judge whether the drop is in focus or not; however, the system needs a specialized light source to illuminate raindrops which is usually impossible when using CCTV cameras. Garg and Nayar [27] attempted to solve this problem using the velocity-size relationship of raindrops. However, this method does not always work because the width of a rain streak in the image appears to be very small and the estimation can be severely influenced by image noise and background clutter. In this paper, the photometric difference between focused and defocused raindrops is studied in-depth and a new algorithm is developed using average color tensor response and average intensity difference of raindrops for identification of different raindrops.

The paper is organized as follows. In Section 2, the proposed algorithm is presented. In Section 3, a rain detection method using gray-tone functions and the constraint of rainfall direction is introduced. In Section 4, the photometric difference between the focused and defocused raindrops is explored, where features are used for discriminating these raindrops. In Section 5, we firstly discuss how to generate a

This work was supported by the National Natural Science Foundation of China (No. 61401239), Project of Industry and School and Research Institution of Jiangsu Province (No.BY2016075-01), Key Support Project of National Natural Science Joint Foundation of China (No. U1613217). The corresponding author is Rong Dong, drwistaria@163.com 
RSD curve from the measurements, and then present a method for measuring rain rates from the fitted RSD curve. Experimental results are shown in Section 6 and conclusions are drawn in Section 7.

\section{DETECTION OF RAINDROPS}

It has been known that raindrops may refract light from the ambient environment, and therefore a raindrop tends to be much brighter than the background that it occludes [18]. On the other hand, the speed of raindrops is so fast that usually a raindrop does not occupy the same image area in two consecutive image frames. Fig. 2 illustrates the intensity changes of a pixel in a rain video, which is temporally fluttering. Based on the above observation, candidate raindrops in the image can be selected using the rules below:

$$
I^{t}-I^{t-1}>I_{t h} \quad \& \quad I^{t}-I^{t+1}>I_{t h}
$$

where $I^{t-1}, I^{t}$, and $I^{t+1}$ are intensity values at the same pixel of three consecutive image frames. This method does not work well as it has a congenital defect due to the arithmetic operations for real numbers [28]. In our approach, the gray tone functions in the LIP framework are used to detect candidate raindrops.

\section{A. Gray Tone functions}

LIP was introduced by $[29,30]$ as a framework for representation and processing of intensity images with values bounded in a certain range. The LIP theory was consistent with human brightness perception. This theory has been used in several image processing areas such as edge detection, image segmentation, and image enhancement [31, 32].

In the LIP framework, an intensity image is represented by its associated gray-tone function $G(x, y)$, which is related to the incident light intensity function $I(x, y)$ by Eq. (2).

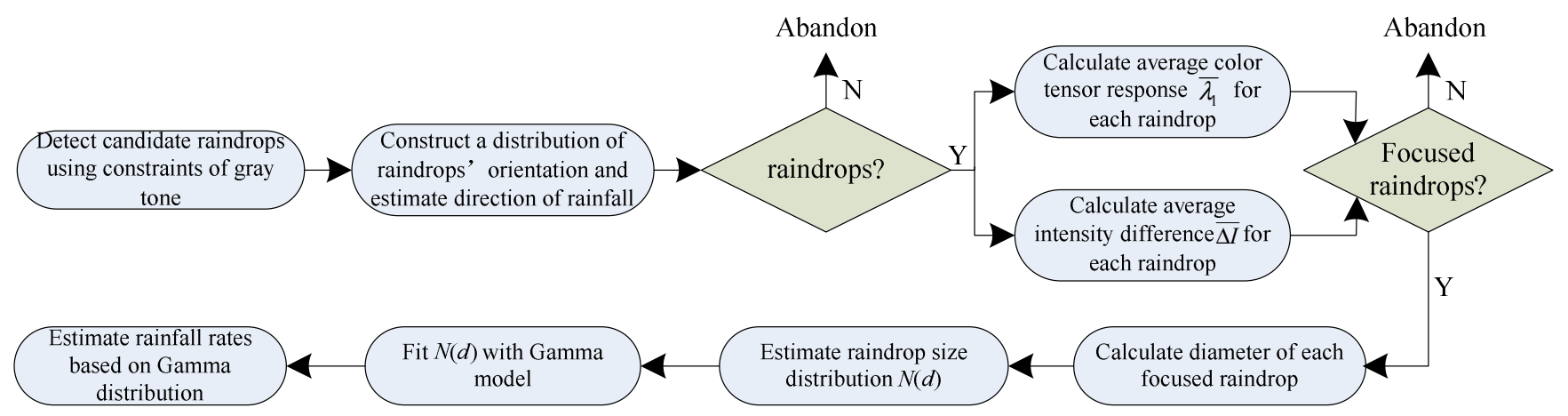

Fig.1. Diagram of proposed algorithm

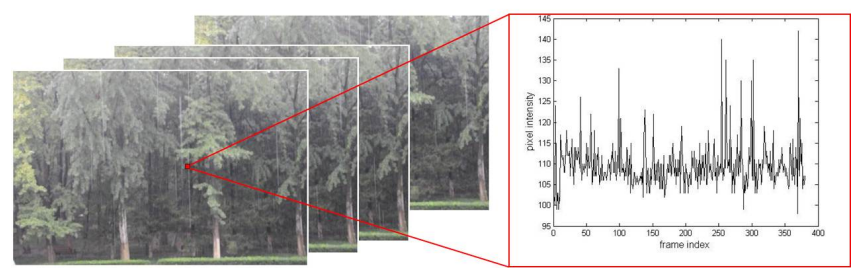

Fig. 2. Intensity changes of a pixel in a rain video acquired by a stationary camera.

$$
G(x, y)=M\left(1-\frac{I(x, y)}{I_{\max }}\right)
$$

$I_{\max }$ is the saturating intensity level in the human visual system. $M$ is the upper bound of gray-tones. Image processing operations such as addition, subtraction and opposite in the LIP framework are defined in a new style. Addition of two gray tone functions $G_{l}(x, y)$ and $G_{2}(x, y)$ is denoted by Eq. (3).

$$
\begin{aligned}
& G_{1}(x, y) \oplus G_{2}(x, y) \\
& =G_{1}(x, y)+G_{2}(x, y)-\frac{G_{1}(x, y) G_{2}(x, y)}{M}
\end{aligned}
$$

It is witnessed that for any two gray-tones in the range [0, $M)$, the result of the addition is still a gray tone in the range $[0, M)$. This is different from the addition operation of intensity values. The opposite (Eq. (4)), subtraction (Eq. (5)) and modulus (Eq. (6)) of the gray-tone functions are respectively defined as:

$$
\begin{gathered}
\Theta G(x, y)=-M \frac{G(x, y)}{M-G(x, y)} \\
G_{1}(x, y) \Theta G_{2}(x, y)=M \frac{G_{1}(x, y)-G_{2}(x, y)}{M-G_{2}(x, y)} \\
|G(x, y)|_{E}=\left\{\begin{array}{cc}
G(x, y) ; & \text { if } G(x, y) \geq 0 \\
\Theta G(x, y) ; & \text { if } G(x, y)<0
\end{array}\right.
\end{gathered}
$$

The subscript $\boldsymbol{E}$ represents the gray-tone space. Since the gray tone function corresponds to the intensity function in an inverted scale, rain candidates are selected using Eqs. (7) and (8):

$$
\begin{gathered}
G^{t}<G^{t-1} \quad \& \quad G^{t}<G^{t+1} \\
\left|G^{t} \Theta G^{t-1}\right|_{E}>G_{t h} \quad \& \quad\left|G^{t} \Theta G^{t+1}\right|_{E}>G_{t h}
\end{gathered}
$$

$G^{t-1}, G^{t}$ and $G^{t+1}$ are the gray-tone functions of three consecutive frames. 
Compared with the use of image intensity, the advantage of using gray-tone is shown in Fig. 3. It is obvious that the results of using gray-tone (Fig. 3(b)) shows clearer rain streaks than that of using normal intensity (Fig. 3(c)). This is because the operation of using gray-tone is nonlinear and relevant to the gray tone itself, while the intensity subtraction is to handle all the intensity values linearly and equally.

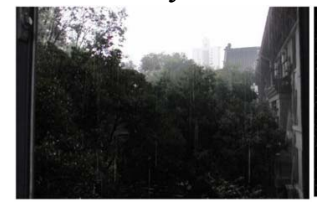

(a)

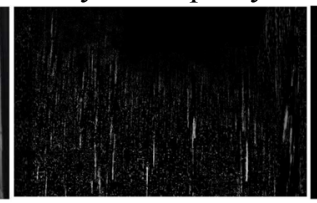

(b)

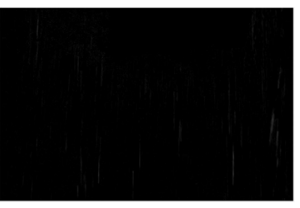

(c)
Fig. 3. Different results of rain candidate detection using the LIP model and using the normal intensity. (a) Current rainy frame. (b) Subtraction of current frame and next frame in the LIP framework; only pixels which satisfy (7) are shown. (c) Result of the same operation but using the corresponding intensity instead of gray tone. $M$ is set to be equal to $I_{\max }=$ 255 in order to operate and display in the same scope.

\section{B. Constraint of Direction}

Rain streaks usually have the same direction as the wind. The constraint of direction may help us remove noise. Finding the tilt angle of a rain streak is equivalent to finding the direction that maximizes the variance of the streak, which can be regarded as the first principal component direction, as in Fig. 4.

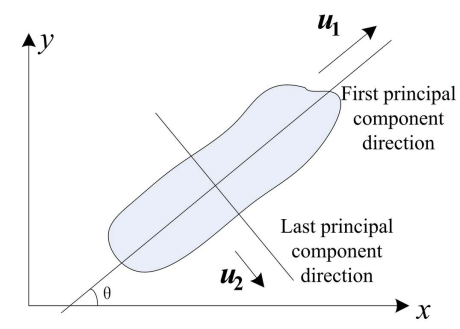

Fig. 4. Principle component directions of a rain streak

Let $N_{i}$ represent the number of the image pixels for a raindrop. $\boldsymbol{X}_{\boldsymbol{i}}$ denotes the $N_{i} * 2$ matrix composed of $N_{i}$ pixel coordinates relative to the center of the streak $i$. Assume $\boldsymbol{u}_{\boldsymbol{i}}{ }^{i}=$ $\left[u_{1 x}{ }^{i}, u_{1 y}{ }^{i}\right]^{\mathrm{T}}, \boldsymbol{u}_{2}{ }^{i}=\left[u_{2 x}{ }^{i}, u_{2 y}{ }^{i}\right]^{\mathrm{T}}$ are eigenvectors that respectively correspond to eigenvalues $\lambda_{1}{ }^{i}, \lambda_{2}{ }^{i}\left(\lambda_{1}{ }^{i}>\lambda_{2}{ }^{i}\right)$ of the covariance matrix $\boldsymbol{X}_{i}^{\boldsymbol{T}} \boldsymbol{X}_{i}$, where $\boldsymbol{u}_{\boldsymbol{i}}^{i}$ is the first principal component direction, and the tilt angle can be calculated as follows:

$$
\theta_{i}=\tan ^{-1}\left(\frac{u_{1 y}{ }^{i}}{u_{1 x}{ }^{i}}\right)
$$

On the other hand, the variances along the two principal component directions can be used to represent the length $a_{i}$ and breadth $b_{i}$ of the streak:

$$
a_{i}=s \sqrt{\frac{\left(\lambda_{1}{ }^{i}\right)^{2}}{N_{i}}}, \quad b_{i}=s \sqrt{\frac{\left(\lambda_{2}{ }^{i}\right)^{2}}{N_{i}}}
$$

where $s$ is a systematic constant.

Considering that the estimation of individual raindrop directions may be disturbed by noise, a distribution of directions $f(\theta)$ is constructed as follows:

$$
f(\theta)=C_{0} \sum_{i} \frac{N_{i}}{\sqrt{2 \pi} h_{i}} k\left(\left\|\frac{\theta-\theta_{i}}{h_{i}}\right\|^{2}\right), k(x)=e^{-\frac{1}{2} x}
$$

where $C_{0}$ is a normalization coefficient. $h_{i}$ is used to describe the uncertain degree of $\theta_{i}$. Consider that the tilt angle is uncertain if the raindrop's length and breadth are close to each other, $h_{i}$ is defined as:

$$
h_{i}=C \frac{b_{i}}{a_{i}}+B
$$

where $C$ is a scale factor to adjust the aspect ratio. $B$ represents a basic bandwidth used in the kernel function.

Notice that Eq. (11) can be viewed as a one-dimension kernel density function. The Mean-shift method [33] is an effective method to determine its local maxima. It is worth noting that bandwidth $h_{i}$ changes with $i$, so the derived Meanshift vector $m(\theta)$ is slightly different from the style shown in [33]:

$$
\begin{gathered}
\nabla f(\theta)=C_{0} \sum_{i} \frac{2 N_{i}}{\sqrt{2 \pi} h_{i}^{3}}\left(\theta_{i}-\theta\right) g\left(\left\|\frac{\theta-\theta_{i}}{h_{i}}\right\|^{2}\right) \\
=\left[C_{0} \sum_{i} \frac{2 N_{i}}{\sqrt{2 \pi} h_{i}^{3}} g\left(\left\|\frac{\theta-\theta_{i}}{h_{i}}\right\|^{2}\right) \times m(\theta)\right. \\
m(\theta)=\frac{\sum_{i} \frac{2 N_{i}}{\sqrt{2 \pi} h_{i}^{3}} \theta_{i} g\left(\left\|\frac{\theta-\theta_{i}}{h_{i}}\right\|^{2}\right)}{\sum_{i} \frac{2 N_{i}}{\sqrt{2 \pi} h_{i}^{3}} g\left(\left\|\frac{\theta-\theta_{i}}{h_{i}}\right\|^{2}\right)}-\theta, g(x)=-k^{\prime}(x)
\end{gathered}
$$

The local maxima $\theta_{\text {main }}$ can be estimated as follows:

Step1: Set an initial $\theta_{0}$;

Step2: Let $\theta_{1}=m\left(\theta_{0}\right)+\theta_{0}$;

Step3: If $\left|\theta_{1}-\theta_{0}\right|<\varepsilon$, stop and return $\theta_{\text {main }}=\theta_{1}$; else let $\theta_{0}=\theta_{l}$ and then go to step1.

Given a local maxima $\theta_{\text {main }}$ of the distribution, which is the most probable rainfall direction in the image, the pixel with an orientation deviation from $\theta_{\text {main }}$ of a threshold larger than $\theta_{t h}$ is considered as noise and should be rejected:

$$
\left|\theta_{j}-\theta_{\text {main }}\right|>\theta_{t h}
$$

\section{DISCRIMINATION OF FOCUSED AND DEFOCUSED RAINDROPS}

The raindrops detected by the framework described above may contain both focused and defocused raindrops. In order to eliminate the defocused raindrops, further processing is applied. Considering a visible point $\mathrm{P}$ in the scene, if it is not in focus, its corresponding image appears to be a blurred circle. According to geometric optics, the blurred image of point $\mathrm{P}$ is actually related to the point spread function of the camera system, and the observed image is the result of convolving the focused image with the camera's point spread function [34]. A simple spread function is: 


$$
h(x, y)= \begin{cases}\frac{4}{\pi d_{c}^{2}} & \text { if } x^{2}+y^{2} \leq d_{c}^{2} / 4 \\ 0 & \text { otherwise }\end{cases}
$$

where $d_{c}$ is the diameter of the blurred circle. It has been shown that the intensity values of a rain streak depend on the brightness of the stationary raindrop as well as the background's radiance and the integration time of the used camera [18]:

$$
I_{r}(x, y)=\int_{0}^{\tau} E_{r}(x, y) d t+\int_{\tau}^{T} E_{b}(x, y) d t
$$

where $\tau$ is the time when the image pixel is occluded by a raindrop. $T$ is the exposure time of the camera. $E_{r}$ is the irradiance due to the raindrop itself and $E_{b}$ is the irradiance due to the background. If the raindrop is not in focus, the irradiance that the image plane receives due to the raindrop is described by:

$$
\begin{aligned}
E_{r}^{\prime}(x, y) & =E_{r}(x, y) * h(x, y) \\
& =\int_{-\infty}^{\infty} \int_{-\infty}^{\infty} E_{r}(x-u, y-v) h(u, v) d u d v \\
& =\frac{4}{\pi d_{c}^{2}} \iiint_{u^{2}+v^{2} \leq d_{c}^{2} / 4} E_{r}(x-u, y-v) d u d v \\
& =E_{r}(x, y) \frac{S_{r}}{S_{d}}
\end{aligned}
$$

where $S_{r}$ is the area of the overlapped region of the raindrop and the corresponding area of the used point spread function. $S_{d}=\pi d_{c}^{2} / 4$ is the area of the point spread function. The convolution process shown in Eq. (18) is illustrated in Fig. 5. Note that $E_{r}{ }^{\prime}(x, y)$ decreases as $d_{c}$ increases. If a raindrop is further away from the focus plane, $d_{c}$ is larger and $E_{r}^{\prime}(x, y)$ is lower. When $d_{c}$ is large enough, we have $S_{r}<S_{d}$. Then:

$$
E_{r}^{\prime}(x, y)<E_{r}(x, y)
$$

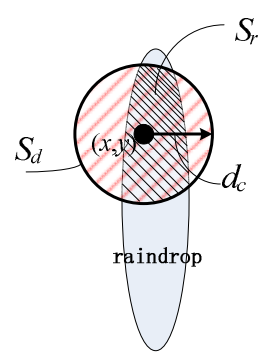

Fig. 5. Illustration of the convolution process of a defocused raindrop. When $d_{c}$ is large, the area $S_{d}$ of the point spread function is bigger than the overlap area $S$, so the convolution process reduces the irradiance $E_{r}(x, y)$
In Eq. (17), $E_{b}$ and $E_{r}$ are assumed to be constant over the entire exposure time $T$. Therefore

$$
I_{r}(x, y)=\tau E_{r}(x, y)+(T-\tau) E_{b}(x, y)
$$

Considering the defocus effect, Eq. (20) can be modified to be:

$$
I_{r}^{\prime}(x, y)=\tau E_{r}^{\prime}(x, y)+(T-\tau) E_{b}(x, y)
$$

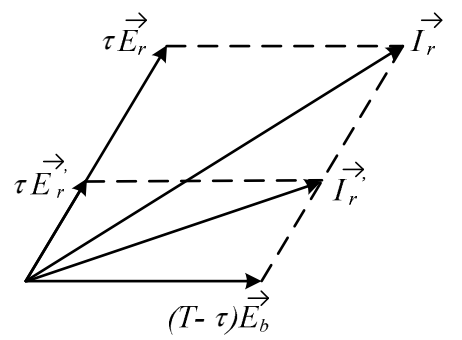

Fig. 6. Comparison of color vectors of focused and defocused raindrops

Eqs. (20) and (21) can be extended to an application in the color space as shown in Fig. 6, where $\vec{I}_{r}$ refers to $\left(I_{r}{ }^{R}, I_{r}{ }^{G}, I_{r}{ }^{B}\right)$, and $\overrightarrow{E_{r}}$ stands for $\left(E_{r}{ }^{R}, E_{r}{ }^{G}, E_{r}{ }^{B}\right)$. In Fig. 6, two principles can be applied to help discriminate the focused and defocused raindrops:

1) The focused raindrops have higher $E_{r}^{\prime}$ than the defocused ones. However, it is difficult to calculate the value of $E_{r}$ r because time $\tau$ is unknown. Note that in Eq. (21), $\tau$ is very small, and $E_{r}^{\prime} \gg E_{b}$, so $\tau E_{b}$ can be ignored. Therefore, a high $E_{r}{ }_{r}$ also indicates a high intensity difference $\Delta I(x, y)=I_{r}^{\prime}(x, y)-I_{b}(x, y)=I_{r}^{\prime}(x, y)-T E_{b}(x, y)=$ $\tau E_{r}^{\prime}$.

According to [27], background intensity $I_{b}(x, y)$ can be restored using two consecutive frames, and therefore $\Delta I$ can be estimated by:

$$
\Delta I=I_{r}^{t}-\left(I_{r}^{t+1}+I_{r}^{t-1}\right) / 2
$$

To make the feature more robust, $\overline{\Delta I}$, which is the average $\Delta I$ of a raindrop, is used as the first feature that allows the focused and defocused raindrops to be discriminated.

2) The color vector distance between the defocused raindrop and its background is smaller than that between the focused raindrop and its background. The edge of the focused raindrop in color space is sharper than that of the defocused one. In order to describe the intensity of an edge in color space, we use a color tensor [35]. Assuming a color image $\boldsymbol{I}=\left(I^{R}, I^{G}, I^{B}\right)$, we have a color tensor defined as:

$$
F=\left(\begin{array}{ll}
\overline{\boldsymbol{I}_{x} \cdot \boldsymbol{I}_{x}} & \overline{\boldsymbol{I}_{x} \cdot \boldsymbol{I}_{y}} \\
\overline{\boldsymbol{I}_{y} \cdot \boldsymbol{I}_{x}} & \overline{\boldsymbol{I}_{y} \cdot \boldsymbol{I}_{y}}
\end{array}\right)
$$


where the symbol ${ }^{-}$indicates the convolution of the color image with a Gaussian filter, and the subscript $x$ or $y$ indicates the spatial derivative along the $x$ or $y$ axis. The largest eigenvalue $\lambda_{l}$ of $F$ represents the derivative energy in the most prominent direction which is used to describe the magnitude of an edge in the color space. Fig. 7(c) shows the $\lambda_{I}$ response of the image shown in Fig. 7(a) $\left(\lambda_{l}\right.$ is calculated only at the region of the detected raindrops shown in Fig. 7(b)). It is noticed that the response of the focused raindrop is greater than that of the defocused one.

To make the feature more robust, $\bar{\lambda}_{1}$, which is the average $\lambda_{I}$ response around the boundary of the raindrop, is used as the second feature that enables the focused and defocused raindrops to be separated.

Fig. 8 shows the distribution of $\overline{\Delta l}$ and $\overline{\lambda_{1}}$ obtained from 25 consecutive images of a rainy scene. The blue asterisks represent the defocused raindrops and the red ones represent the focused ones. The focused raindrops are chosen manually. It is observed that the focused raindrops lie mainly in the first quadrant of the green coordinate system, indicating that the two parameters $\overline{\Delta I}$ and $\bar{\lambda}_{1}$ can be used to discriminate the focused and the defocused rain streaks properly. Therefore, for every detected raindrop, $\overline{\Delta l}$ and $\bar{\lambda}_{1}$ are calculated and then used to determine whether the raindrop is in focus or not. In other words, if both of the two parameters are higher than a certain threshold, this raindrop is classified as a focused one.

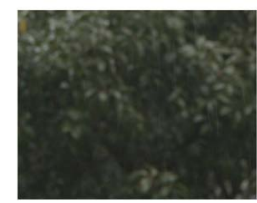

(a)

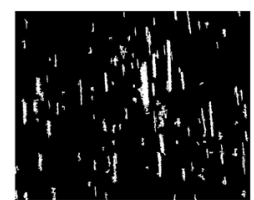

(b)

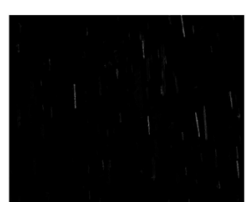

(c)
Fig. 7: Detected rain drops in a frame and their $\lambda_{l}$ response: (a) Current Frame. (b) Detected raindrops. (c) $\lambda_{I}$ response of the current frame.

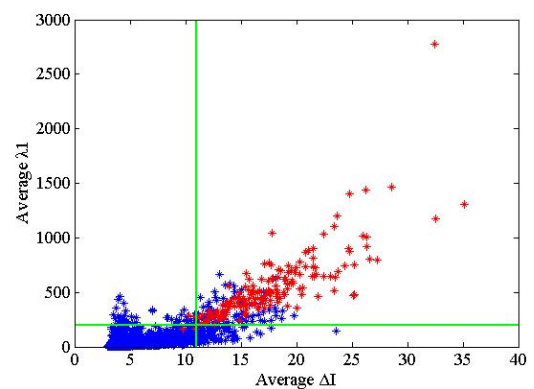

Fig. 8. Distribution of $\overline{\Delta l}$ and $\bar{\lambda}_{1}$ obtained from 25 consecutive images of a rainy scene. The blue asterisks represent defocused raindrops and the red ones represent focused ones. Focused raindrops lie mainly in the first quadrant of the green coordinate system. Therefore, focused and defocused rain streaks can be discriminated using the properly defined $\overline{\Delta l}$ and $\overline{\lambda_{1}}$.

\section{MEASUREMENT OF RAINFALL RATES}

The number and the size of raindrops within a unit volume and a unit scale interval can be described by the number concentration $N(d)\left[\mathrm{m}^{-3} \mathrm{~mm}^{-1}\right]$, also called RSD, where $d[\mathrm{~mm}]$ is the equivalent spherical diameter of a raindrop. Once a focused rain streak in the image has been detected, its length $a_{i}$ is calculated using a method of geometric moments presented in [36]. Considering a rain streak $i$ with depth $z$ and length $a_{i}$, and using an affine transformation, we have:

$$
\frac{z}{f}=\frac{T \cdot v(d)}{a_{i}}
$$

where $T$ is the time of exposure. The relationship between the diameter of a raindrop and its terminal speed $(\mathrm{m} / \mathrm{s})$ is [37]:

$$
v(d)=3.778 d^{0.67}
$$

The diameter $d_{i}$ can be calculated by:

$$
d_{i}=\left(\frac{z a_{i}}{3.778 f T}\right)^{\frac{1}{0.67}}
$$

According to the definition of RSD, $N(d)$ is obtained by firstly counting the number of raindrops which have diameters falling in the range $[d-1 / 2 \Delta d, d+1 / 2 \Delta d]$, where $\Delta d$ is the unit scale interval $(1 \mathrm{~mm})$. Then we divide this statistical result by the space volume $V$, which is approximated by the product of the depth of field and the width and height of the scene captured at the focal plane. In order to obtain a correct result, the counting process is implemented over multiple consecutive frames.

In meteorological science, there are several well-known RSD models, such as Exponential distribution, Gamma distribution and M-P distribution [38]. It has been shown that Gamma distribution can produce good performance for different kinds of precipitation and is hence widely used [39]. In this paper, we use a Gamma distribution model to fit the RSD curve obtained and then calculate rainfall rates from the model. The Gamma distribution is defined as:

$$
N(d)=N_{0} d^{\mu} e^{-\omega d}
$$

where $N_{0}, \mu$ and $\omega$ are three parameters which can be solved by a moment method [40]. The ith order moment of the RSD is defined as:

$$
M_{i}=\int_{0}^{\infty} d^{i} N(d) d d
$$

Substituting $N(d)$ into Eq. (28) with the Gamma distribution in (27), then we have:

$$
M_{i}=N_{0} \frac{\Gamma(i+\mu+1)}{\omega^{i+\mu+1}}
$$

$N_{0}, \mu$ and $\omega$ are respectively calculated by: 


$$
\mu=\frac{3 M_{4} M_{2}-4 M_{3}^{2}}{\left(M_{3}^{2}-M_{4} M_{2}\right)}, \omega=\frac{(\mu+4) M_{3}}{M_{4}}, \quad N_{0}=\frac{\omega^{\mu+4} M_{3}}{\Gamma(\mu+4)}(30)
$$

According to [37], finally, a rainfall rate is given by:

$$
R=\frac{6 \pi}{1000} \int_{0}^{\infty} N(d) v(d) d^{3} d d=0.0071 N_{0} \frac{\Gamma(\mu+4.67)}{\omega^{\mu+4.67}}
$$

\section{EXPERIMENT RESULTS}

The proposed algorithm was tested on various rainy days with light, middle or heavy rain at Nanjing City in China. Rainfall rates calculated were compared against the ground truth data measured using a can-type rain gauge. The rain gauge was used to collect rainwater during a certain time interval and then the volume of rain was converted to the standard rainfall rate values in $\mathrm{mm} / \mathrm{h}$. The videos were taken by a SONY DSR-PD198P video camera.

\section{A. Camera parameters adjustment}

In order to get a good size distribution curve of a raindrop, the smallest raindrop should be able to be captured in the image. To do so, the focal length and the focal plane of the camera must be adjusted properly. As shown in Fig. 9, the smallest raindrop should have occupied at least one pixel so as to be clearly visible. Thus we have:

$$
z \leq f d_{\min }
$$

In the experiment, we observe that the focused raindrops can be segmented better as $z$ becomes smaller. This is because if the focal plane is further away from the camera, there will be more defocused raindrops overlapping the focused ones, which make it more difficult to segment the focused raindrops from their counter parts. In all the experiments, we empirically set $z=1(\mathrm{~m})$.

When $z$ is determined, $f$ has a lower limit according to Eq. (32). In fact, there is also an upper limit for $f$. The scene captured by the camera should be large enough so that the largest raindrop can be detected. This suggests that the height of the image should be larger than the length of the longest rain streak. Suppose that $d_{\max }$ is the diameter of the largest raindrop; then $f$ must satisfy this equation:

$$
f \leq \frac{z H}{T \times v\left(d_{\text {max }}\right)}
$$

where $H$ is the height of the image, and $v\left(d_{\max }\right)$ is calculated by Eq. (25).

Using the variables determined by Eqs. (32) and (33), we manually (or by remote control) zoom in the camera view when the rain is light, and zoom out when the rain is heavy. Large raindrops (e.g. the diameter is greater than $5 \mathrm{~mm}$ ) appear in heavy rain conditions, and small raindrops (e.g. the diameter is smaller than $3 \mathrm{~mm}$ ) show up when rain is light. The aperture $A$ of the camera is accordingly adjusted in order for us to obtain a small depth of field.

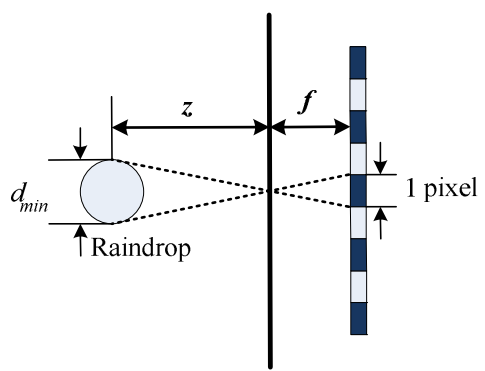

Fig. 9. The smallest raindrop should be visible in the image. This means that the smallest raindrop occupies at least one image pixel in the image.

\section{B. Rain detection and focused raindrops identification}

Typical images captured and the corresponding detection results are shown in Fig. 10. The first row shows typical images corresponding to light, moderate and heavy rain situations respectively. Notice that we manually zoom in the camera view when the rain is light, and zoom out when the rain is heavy, so the background scenery in a heavy rain situation looks sharper than that of light rain. Individual parameter settings of these three situations are listed in the next section.

Candidate raindrops detected using gray-tone functions are shown in the second row. Raindrops can be detected correctly. Due to noise and background variation, a small amount of false detections occurs. Constructed distributions of rainfall directions from the binary images of candidate raindrops are shown in the third row. Using the proposed mean-shift method, we detect the local maxima of the distribution function $\theta_{\text {main }}$. The raindrop with an orientation deviating by more than $\theta_{t h}$ from $\theta_{\text {main }}$ is considered as noise and will be rejected. In all the experiments, $\theta_{t h}$ is empirically set to be 10 , while the scale factor $C$ and the basic bandwidth $B$ are respectively set to be 30 and 5. The detected raindrops using the constraint of direction are shown in the fourth row. The algorithm proposed in Section 4 is used to further discriminate the focused and defocused raindrops and finally the focused raindrops are shown in the fifth row. At this stage, two threshold values corresponding to average color tensor response $\bar{\lambda}_{1}$ and average intensity difference $\overline{\Delta l}$ respectively affect the final result. Considering different illumination conditions in different rainy situations, the two threshold values are trained in advance. We manually select the focused and defocused raindrops from a section of the training sequence and construct a distribution map of $\overline{\Delta I}$ and $\bar{\lambda}_{1}$ shown in Fig. 8, then determine the threshold values.

\section{Rainfall rate measurement}

The measured $N(d)$ and the fitted Gamma distribution model are shown in Fig. 11. The counting process is implemented using 1000 consecutive frames. This means that the system outputs an "instantaneous rain rate" every 1000 frames. Detailed parameters settings are shown in Table 1. The mean rainfall rate is calculated by averaging instantaneous rain rates during a period of time (e.g. half an hour for moderate rain and the length of time depends on the rain situation at that day). It can be observed that although there are minor disturbance, the measured RSD curves under 
different rainy conditions are consistent with those of the Gamma distributions. It is worth pointing out that under heavy rainy conditions the density of large raindrops calculated using our approach is higher than the fitted Gamma distribution. This is mainly because the occlusion between two raindrop streaks becomes severe when the rain is heavy and the two short streaks may join together to form a longer one, which is difficult to separate.

In Table 1, we show that the calculated rainfall rates also agree with the records of the can-type pluviometer. The two results are not exactly the same because both methods have errors. The systematic errores of the can-type pluviometer mainly include: wetting loss caused by the wetting of the inner walls of both collector and container, wind-caused loss because of wind preventing small raindrops from entering the gauge and out-splashing loss caused by collision between raindrops and the inwall of the collector. The total magnitude of its systematic errors may be up to $15 \%$ [41]. Besides, reading error exists because the can-type rain gauge is a manual one. Most errors of can-type rain gauge are loss-type, that's maybe why the meansurement values by proposed algorithm are larger in moderate and heavy rain situations. In the light rain situation, our meansurement result appears to be lower by contrast with the can-type. That's maybe because most rain drops are very small in light rain so that their rain streaks are very slender and their image intensities are low which may cause more streaks' break and miss detection. Despite the minor difference, these results show that our algorithm is capable of obtaining reasonably correct rainfall rates.

TABLE 1 PARAMETER SETTINGS AND MEASUREMENT RESULTS

\begin{tabular}{llll} 
Weather condition & Light rain & Moderate rain & Heavy rain \\
\hline$f$ & 5373 & 4179 & 3383 \\
$A$ & 2.8 & 2.6 & 2.6 \\
$T[\mathrm{~s}]$ & $1 / 215$ & $1 / 215$ & $1 / 215$ \\
$z[\mathrm{~m}]$ & 1 & 1 & 1 \\
$\begin{array}{l}\text { Rainfall rate by } \\
\text { pluviometer }[\mathrm{mm} / \mathrm{h}]\end{array}$ & 1.1 & 3.5 & 6.5 \\
$\begin{array}{l}\text { Mean Rainfall rate } \\
\text { by presented } \\
\text { algorithm }[\mathrm{mm} / \mathrm{h}]\end{array}$ & 0.8 & 4.7 & 8.7 \\
\hline
\end{tabular}

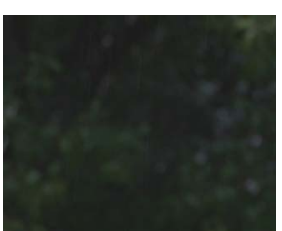

(a)

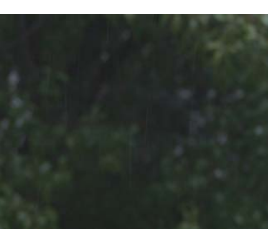

(b)

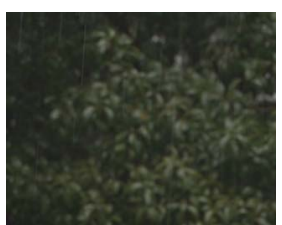

(c)

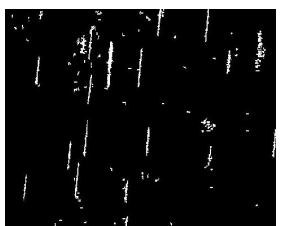

(d)

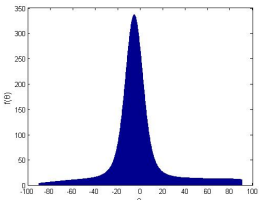

(g)

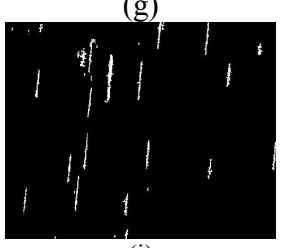

(j)

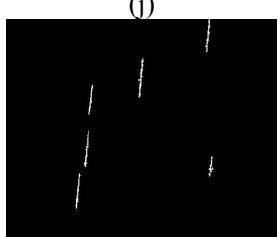

(m)

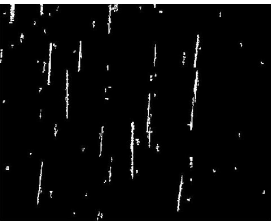

(e)

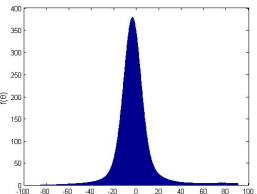

(h)

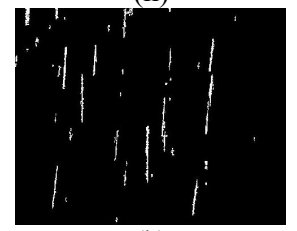

(k)

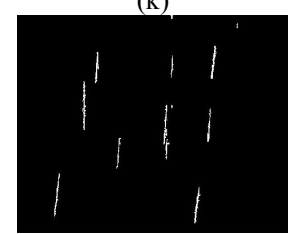

(n)

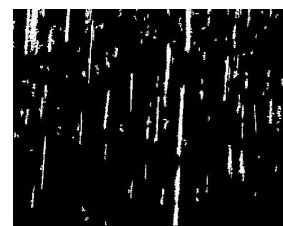

(f)

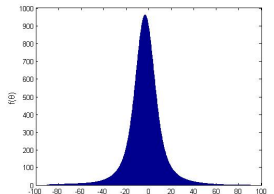

(i)
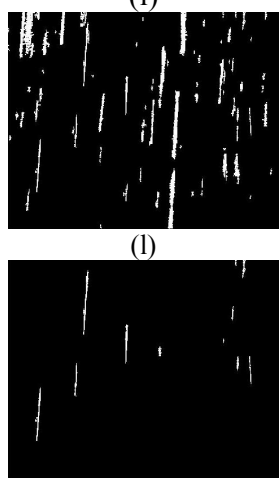

(o)
Fig. 10: Typical images captured and corresponding detection results. First row: typical images corresponding to light rain, moderate rain and heavy rain situations respectively. Second row: candidate raindrops detected using constraints of gray tone. Third row: constructed distribution of orientations. Fourth row: raindrops detected after constraints of direction. Fifth row: finally identified focused raindrops.

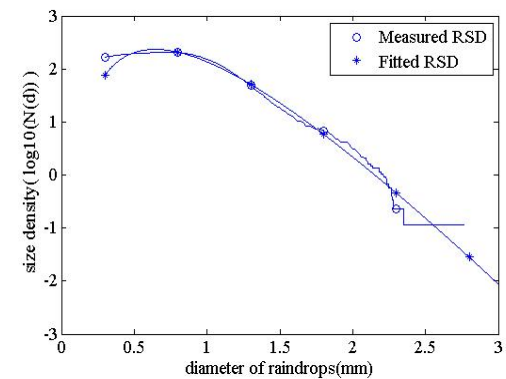

(a)

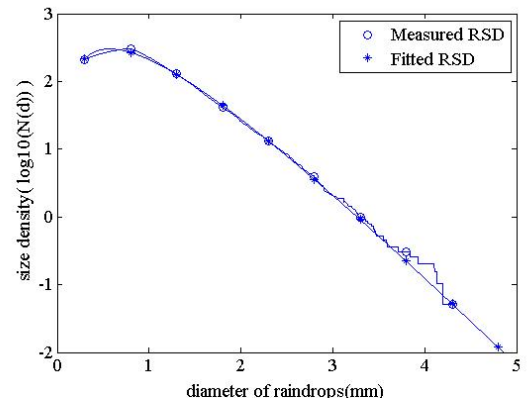

(b) 


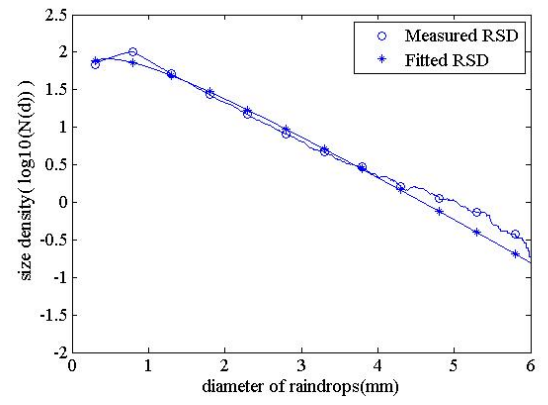

(c)

Fig. 11: Measured RSD curve and fitted Gamma distribution under different rainy conditions: (a) light rain, (instantaneous rain rate 1.0 $\mathrm{mm} / \mathrm{h}$ ). (b) moderate rain, (instantaneous rain rate $4.9 \mathrm{~mm} / \mathrm{h}$ ). (c) heavy rain, (instantaneous rain rate $11.0 \mathrm{~mm} / \mathrm{h}$ ).

\section{CONCLUSIONS}

In this paper, a method for real-time rainfall rate measurement has been presented. The method we proposed is to count the focused raindrops in a small depth of field and then create a RSD curve. Afterwards, rainfall rates can be estimated by fitting the curve using a Gamma distribution model. To achieve this target, accurate detection of raindrops is the first task, followed by the discrimination of focused and defocused raindrops. Detecting raindrops using gray-tone functions demonstrated the capability to identify raindrops better than using image intensities. The constraint of direction helps to remove false detections. It has shown that average color tensor response and average intensity difference of raindrops can be used to discriminate the focused and defocused raindrops in the images. The effectiveness of our algorithm has been demonstrated in the experiments. The weakness of the proposed algorithm is that rainfall rate measurements from videos may be less accurate than those of rain gauges. This is because of several inevitable errors such as the precision of the estimated raindrop sizes and space volumes. In spite of this weakness, the proposed method paves the way to estimate rainfall rates from videos automatically.

\section{REFERENCES}

[1] E. Habib, W. F. Krajewski, and A. Kruger, "Sampling errors of tipping bucket rain gauge measurements," J. Hydrol. Eng., vol.6, no.2, pp.159-166, 2001.

[2] S. Michaelides, Precipitation: Advances in Measurement, Estimation and Prediction, Berlin Heidelberg, Springer-Verlag, 2008.

[3] V. N. Bringi and V. Chandrasekar, Polarimetric Doppler Weather Radar, Cambridge University Press, 2001.

[4] B. E. Sheppard, "Measurement of raindrop size distributions using a small doppler radar," J. Atmos. Oceanic Technol., vol.7, no.2, pp.255-268, 1990.

[5] G. Peters, B. Fischer, H. Münster, et al., "Profiles of raindrop size distributions as retrieved by microrain radars," J. Appl. Meteorol., vol.44, no.12, pp.1930-1949, 2005.
[6] T. J. Mansheim, A. Kruger, J. Niemeier, and A. J. B. Brysiewicz, “A robust microwave rain gauge," IEEE Trans. Instrum. Meas., vol.59, no.8, pp.2204-2210, 2010.

[7] M. Loffler-Mang and J. Joss, "An optical disdrometer for measuring size and velocity of hydrometeors," J. Atmos. Oceanic Technol., vol.17, no.2, pp.130-139, 2000.

[8] R. P. Lawson, D. O'Connor, P. Zmarzly, et al., "The 2D-S (stereo) probe: design and preliminary tests of a new airborne, high-speed, high-resolution particle imaging probe," J. Atmos. Oceanic Technol, vol.23, no.11, pp.1462-1477, 2006.

[9] H. Minda and N.Tsuda, "Low-cost laser disdrometer with the capability of hydrometeor imaging," IEEJ T. Electr. Electr., vol.7, no.S1, pp.S132-S138, 2012.

[10] A. Kruger, W. F. Krajewski, "Two-dimensional video disdrometer: a description," J. Atmos. Oceanic Technol., vol.19, no.5, pp.602617,2002

[11] M. Schonhuber, G. Lammer, W. L. Randeu, "One decade of imaging precipitation measurement by 2D-video-distrometer," $A d v$. Geosci., vol.10, pp.85-90, 2007.

[12] K. Suzuki, K. Shimizu, T. Ohigashi, et al., "Development of a new videosonde observation system for in-situ precipitation particle measurements," SOLA, vol.8, pp.001-004, 2012.

[13] G. Frank, T. Hartl, J. Tschiersch, "The pluviospectrometer: classification of falling hydrometeors via digital image processing," Atmos. Res., vol.34, pp.367-378, 1994.

[14] J. R. Saylor and N. A. Sivasubramanian, "Edge detection methods applied to the analysis of spherical raindrop images," Appl. Opt., vol.46, no.22, pp.5352-5367, 2007.

[15] X. C. Liu, T. C. Gao, and L. Liu, "A video precipitation sensor for imaging and velocimetry of hydrometeors," Atmos. Meas. Tech., vol.7, pp.2037-2046, 2014.

[16] S. Shirmohammadi and A. Ferrero, "Camera as the instrument: the rising trend of vision based measurement," IEEE Instru. Meas. Mag., vol.17, no.3, pp.41-47, 2014.

[17] A. K. Tripathi and S. Mukhopadhyay, "Removal of rain from videos: a review," Signal Image Video P., vol.5, pp.1-10, 2012.

[18] K. Garg and S. K. Nayar, "Detection and removal of rain from videos," In Proc. CVPR, 2004, pp.1:528-535.

[19] X. P. Zhang, H. Li, Y. Y. Qi, W. K. Leow, and T. K. Ng, "Rain removal in video by combining temporal and chromatic properties," In Proc. ICME, 2006, pp.461-464.

[20] P. Liu, J. Xu, J. Liu, X. Tang, "Pixel based temporal analysis using chromatic property for removing rain from videos," Computer and Information Science, vol.2, no.1, pp.53-60, 2009.

[21] Y. Miao, H. Hong, and H. Kim, "Size and angle filter based rain removal in video for outdoor surveillance systems," In Proc. ASCC, 2011, pp.1300-1304.

[22] J. Bossu, N. Hautière, and J.P. Tarel, "Rain or snow detection in image sequences through use of a histogram of orientation of streaks," Int. J. Comput. Vision, vol.93, no.3, pp.348-366, 2011.

[23] A. K. Tripathi and S. Mukhopadhyay, "Video post processing: low latency spatiotemporal approach for detection and removal of rain," IET Image Process., vol.6, no.2, pp.181-196, 2012.

[24] A. K. Tripathi and S. Mukhopadhyay, "Meteorological approach for detection and removal of rain from videos," IET Comput. Vis., vol.7, no.1, pp.36-47, 2013.

[25] J. Chen, L. P. Chau, "A rain pixel recovery algorithm for videos with highly dynamic scene," IEEE Trans. Image Process., vol.23, no.3, pp.1097-1104, 2013.

[26] P. C. Barnum, S. Narasimhan and T. Kanade, "Analysis of rain and snow in frequency space," Int. J. Comput. Vision, vol.86, no.2-3, pp.256-274, 2010.

[27] K. Garg and S. K. Nayar, "Vision and rain," Int. J. Comput. Vision, vol.75, no.1, pp.3-27, 2007.

[28] R. Dong, B. Li, Q. M. Chen, "A method for detection and removal of rain in videos," Acta Automatica Sinica, vol.39, no.7, pp. 10931099, 2013. 
[29] J. C. Pinoli, "The logarithmic image processing model: connections with human brightness perception and contrast estimators," J. Math. Imaging Vis., vol.7, no.4, pp.341-358, 1997.

[30] M. Jourlin and J. C. Pinoli, "Logarithmic image processing: the mathematical and physical framework for the representation and processing of transmitted images," Adv. Imag. Elect. Phys., vol.115, pp.129-196, 2001.

[31] K. Panetta, S. Agaian, Y. C. Zhou, E. J. Wharton, "Parameterized logarithmic framework for image enhancement," IEEE Trans. Syst., Man, Cybern. : Cybern, vol.41, no.2, pp.460-473, 2011.

[32] G. Deng, "A generalized logarithmic image processing model based on the gigavision sensor model," IEEE T. Image Process., vol.21, no.3, pp.1406-1414, 2012.

[33] D. Comaniciu and P. Meer, "Mean shift: a robust approach toward feature space analysis," IEEE Trans. Pattern Anal., vol.24, no.5, pp.603-619, 2002.

[34] M. Subbarao and N. Gurumoorthy, "Depth recovery from blurred edges," In Proc. CVPR, 1988, pp.498-503.

[35] R. Lukac and K. N. Plataniotis, Color Image Processing: Methods and Applications, Boca Raton, Crc Press, 2007, pp.203-225.
[36] R. Safee-Rad, K. C. Smith, B. Benhabib and I. Tchoukanov, "Application of moment and Fourier descriptors to the accurate estimation of elliptical-shape parameters," Pattern Recogn. Lett., vol.13, pp.497-508, 1992.

[37] D. Atlas and C. W. Ulbrich, "Path- and Area-Integrated rainfall measurement by microwave attenuation in the $1-3 \mathrm{~cm}$ band," $J$. Applied meteorology, vol.16, pp.1322-1331, 1977.

[38] C. R. Williams and K. S. Gage, "Raindrop size distribution variability estimated using ensemble statistics," Ann. GeophysGermany, vol.27, no.2, pp.555-567, 2009.

[39] B. J. Chen, Z. H. Li and J. C. Liu, "Model of raindrop size distribution in three types of precipitation," ACTA Meteorologica Sinica, vol.56, no.4, pp.506-512, 1998.

[40] J. H. Zheng and B. J. Chen, "Comparative study of exponential and gamma functional fits to observed raindrop size distribution," Scientia Meteorologica Sinica, vol.27, no.1, pp.17-25, 2007.

[41] B. Sevruk, "Point precipitation measurements: why are they not corrected?" IAHS Publ., no.164 (Water for the Future: Hydrology in Perspective), pp.477-486, 1987. 